\title{
The effects of tumour necrosis factor- $\alpha$ and interleukin 1 on an in vitro model of thyroid-associated ophthalmopathy; contrasting effects on adipogenesis
}

\author{
T J Cawood ${ }^{1,2}$, P Moriarty ${ }^{3}$, C O'Farrelly ${ }^{2,4}$ and D O'Shea ${ }^{1}$ \\ ${ }^{1}$ Department of Endocrinology, and ${ }^{2}$ Education and Research Centre, Saint Vincent's University Hospital, Dublin, Ireland, ${ }^{3}$ Royal Victoria Eye and Ear \\ Hospital, Dublin, Ireland and ${ }^{4}$ The Conway Institute, University College Dublin, Dublin, Ireland
}

(Correspondence should be addressed to T J Cawood; Email: tomjcawood@eircom.net)

\begin{abstract}
Objective: Cytokines are likely to play a key pathogenic role in thyroid-associated ophthalmopathy (TAO). Anti-cytokine therapy has been proposed to be a possible treatment for active TAO. We aimed to establish the effects of selected cytokines on intercellular adhesion molecule 1 (ICAM1) expression, glycosaminoglycan (GAG) production and adipogenesis in orbital fibroblasts (OFs) from patients with TAO. Methods: Orbital tissue was taken during surgery from eight patients with TAO and five control subjects. OFs were cultured and ICAM1 expression measured by flow cytometry. GAG production was measured by hyaluronic acid ELISA. OFs were grown in adipogenic media and the degree of adipogenesis quantified. Results: Responses were similar in OFs from patients with and without TAO. Tumour necrosis factor- $\alpha$ $(\mathrm{TNF} \alpha)$ and interleukin1 (IL1) $(0.1 \mathrm{ng} / \mathrm{ml})$ stimulated ICAM1 expression by eight- to ten-fold. Anticytokine agents inhibited the cytokine-upregulated ICAM1 expression by 90-99\% $(P<0.01)$. TNF $\alpha$ and IL1 $(0.1 \mathrm{ng} / \mathrm{ml})$ increased hyaluronic acid production by 44 and $95 \%(P<0.01)$ respectively. Anti-cytokine agents inhibited these responses by $79-138 \%(P<0.04)$ TNF $\alpha(0.1 \mathrm{ng} / \mathrm{ml})$ inhibited adipogenesis $(-0.013 \mathrm{AU}$ and $-1.0 ; P<0.03)$ whilst IL1 $(0.1 \mathrm{ng} / \mathrm{ml})$ stimulated adipogenesis $(+0.05 \mathrm{AU}$ and $+5.7 ; P<0.02)$ measured by oil-red-O extraction and visual assessment respectively. The anti-IL1 agent inhibited IL1-mediated adipogenesis by $69-106 \%(P<0.04)$.

Conclusion: TNF $\alpha$ and IL1 stimulate ICAM1 expression and GAG production, but have opposite effects on adipogenesis in OFs in vitro. IL1 promotes adipogenesis and its effects can be blocked by anti-IL1 agents in vitro. These agents may be the anti-cytokine treatment of choice for clinical trials in active TAO.
\end{abstract}

European Journal of Endocrinology 155 395-403

\section{Introduction}

Thyroid-associated ophthalmopathy (TAO) is an autoimmune inflammatory condition that is also known as thyroid eye disease and Graves' ophthalmopathy. TAO affects between 25 and $50 \%$ of those with autoimmune hyperthyroidism, with 3-5\% suffering severe eye disease, which may threaten vision $(1,2)$. The pathogenesis is not fully understood, but the key pathological processes include acute inflammation, excess production of glycosaminoglycans (GAG) and expansion of adipose tissues within the orbit (3). The treatment for more severe cases of TAO is often unsatisfactory and there has been increasing interest in the possibility of using anti-cytokine agents to treat active TAO $(4,5)$. This idea is based on in vitro data on the effects of cytokines on orbital fibroblasts (OFs) and the growing experience of the use of anti-cytokine agents in other inflammatory conditions, such as rheumatoid arthritis and inflammatory bowel disease. Cytokines, such as interferon $\gamma($ IFN $\gamma)$, interleukin 1 (IL1) and tumour necrosis factor- $\alpha$ (TNF $\alpha$ ) have been shown to stimulate GAG production from OFs in vitro (6-9). These same cytokines have been shown to increase ICAM1 expression using immunohistochemical techniques (10) and are also increased in active TAO in vivo (11).

These and other data suggest that blocking these cytokines might inhibit some of the pathogenic processes in TAO, including the orbital inflammatory response and excess GAG production, therefore limiting orbital tissue expansion. However, there are data that show that $\mathrm{TNF} \alpha$ can inhibit orbital adipogenesis, and hence blocking $\mathrm{TNF} \alpha$ might have a harmful effect on TAO by increasing adipogenesis and increasing orbital tissue volume (12).

We aimed to evaluate the effects of selected cytokines and anti-cytokine agents on cultured OFs as an in vitro model of TAO. We focused on TNF $\alpha$ and IL1, because anticytokine agents that block the action of these cytokines are presently in clinical use for indications other than TAO. We chose to examine three endpoints: ICAM1 expression, GAG production and adipogenesis. Increased ICAM1 expression plays a role in inflammatory cell 
recruitment and occurs in active TAO (11). Thus, a reduction in ICAM1 expression would be expected to have an inhibitory effect on the local inflammatory response. We measured GAG production by orbital fibroblasts and adipogenesis in pre-adipocyte orbital fibroblasts, as these processes are thought to cause tissue swelling in TAO $(1,13)$.

\section{Subjects}

TAO was diagnosed on clinical grounds based on the presence of typical clinical features in the context of autoimmune thyroid disease. In the TAO group, seven out of the eight patients were female, median age 49 years (range 42-65), median Mourits activity score was 1 (range 0-9), and five of the eight patients were smokers (Table 1). Control subjects were patients without a personal or family history of thyroid disease or TAO, and without clinical evidence of the same, who were attending orbital surgery. In the control group, four out of the five patients were female, median age 48 years (range $21-77$ ) and all were non-smokers (Table 1). The study was approved by the institutional ethics committees and all study participants gave written informed consent.

\section{Methods}

\section{Orbital fibroblast cultures}

Orbital fibroblast (OF) cultures were established, similar to published methods (14). Orbital fat biopsies (approximately 4-8 $\mathrm{mm}$ in length) were taken at surgery and transported to the laboratory in normal saline at room temperature. The biopsies were diced using a scalpel blade and placed in RPMI tissue culture medium containing $9 \%$ fetal bovine serum, $20 \mathrm{ml} / \mathrm{l}$ of $1 \mathrm{M}$ HEPES, $1.8 \mathrm{mg} / \mathrm{l}$ fungizone, $35 \mathrm{U} / \mathrm{ml}$ penicillin and $35 \mu \mathrm{g} / \mathrm{ml}$ streptomycin in a $25 \mathrm{~cm}^{2}$ flask and grown at $37{ }^{\circ} \mathrm{C}$ in $5 \% \mathrm{CO}_{2}$ and $21 \% \mathrm{O}_{2}$ (all additives from Gibco, Invitrogen). Once OFs were adherent, the flask was washed with culture media and OFs grown to confluence replacing media every 3-4 days. Confluent fibroblasts were passaged and plated into new flasks or multi-well plates, with typically 20000 cells being seeded into each well of a 12-well plate, which could be grown to confluence in 5-10 days.

Cytokines (TNF $\alpha$, IL1, IFN $\gamma$, transforming growth factor- $\beta$ (TGF $\beta$ ) and IL10) were purchased from R\&D Systems and the concentrations chosen from logarithmic dose-response experiments, they being on either side of the ED50. The concentrations of anti-cytokine agents used were based upon published levels of peak serum concentrations in humans following typical therapeutic doses (anti-TNF $\alpha$ agents adalimumab $50 \mu \mathrm{g} / \mathrm{ml}(15)$, infliximab $150 \mu \mathrm{g} / \mathrm{ml}$ (16) and etanercept $2.5 \mu \mathrm{g} / \mathrm{ml}(17)$ and anti-IL1 agent anakinra $5 \mu \mathrm{g} /$ $\mathrm{ml}(18)$ ), and a concentration $1 / 100$ of the same.

\section{ICAM1 measurements}

The expression of ICAM1 by OFs was analyzed using flow cytometry. OFs were grown to confluence in 12-well plates. Confluent OFs were given fresh medium and stimulated for 24 or $48 \mathrm{~h}$ with the cytokine \pm anticytokine agent, with un-stimulated wells as control. OFs were trypsinized and centrifuged, and resuspended in $200 \mu \mathrm{l}$ of culture medium. OFs were then added to $5 \mu \mathrm{l}$ of

Table 1 Patient details.

\begin{tabular}{|c|c|c|c|c|c|c|c|}
\hline Sex & $\begin{array}{c}\text { Age } \\
\text { (years) }\end{array}$ & $\begin{array}{l}\text { Duration of } \\
\text { TAO (years) }\end{array}$ & $\begin{array}{c}\text { Clinical } \\
\text { activity score }^{a}\end{array}$ & $\begin{array}{l}\text { Cigarette } \\
\text { smoker }\end{array}$ & $\begin{array}{l}\text { Previous orbital } \\
\text { radiotherapy }\end{array}$ & $\begin{array}{l}\text { Previous or current } \\
\text { steroids for TAO }\end{array}$ & Surgical procedure \\
\hline \multicolumn{8}{|c|}{ Controls } \\
\hline M & 53 & $\mathrm{n} / \mathrm{a}$ & 0 & No & No & $\mathrm{n} / \mathrm{a}$ & Ptosis repair \\
\hline $\mathrm{F}$ & 48 & $\mathrm{n} / \mathrm{a}$ & 0 & No & No & $\mathrm{n} / \mathrm{a}$ & Ptosis repair \\
\hline $\mathrm{F}$ & 21 & $\mathrm{n} / \mathrm{a}$ & 0 & No & No & $\mathrm{n} / \mathrm{a}$ & Congential ptosis repair \\
\hline $\mathrm{F}$ & 77 & $\mathrm{n} / \mathrm{a}$ & 0 & No & No & $\mathrm{n} / \mathrm{a}$ & Ptosis repair \\
\hline \multicolumn{8}{|l|}{ TAO } \\
\hline $\mathrm{F}$ & 65 & 1.5 & 1 & No & Yes & Yes & Lid recession \\
\hline $\mathrm{F}$ & 47 & 16 & 1 & Yes & No & Yes & Lid recession \\
\hline $\mathrm{F}$ & 53 & 0.8 & 9 & Yes & No & Yes & $\begin{array}{l}\text { Emergency orbital } \\
\text { decompression }\end{array}$ \\
\hline $\mathrm{F}$ & 51 & 0.6 & 2 & Yes & No & No & $\begin{array}{l}\text { Lid recession due to } \\
\text { exposure keratopathy }\end{array}$ \\
\hline$M$ & 42 & 4.5 & 0 & No & Yes & Yes & Orbital decompression \\
\hline $\mathrm{F}$ & 46 & 5 & 0 & Yes & No & Yes & Lid recession \\
\hline $\mathrm{F}$ & 66 & 1 & 5 & Yes & No & Yes & $\begin{array}{c}\text { Emergency orbital } \\
\text { decompression }\end{array}$ \\
\hline
\end{tabular}

n/a, not applicable.

${ }^{a}$ Clinical activity score refers to the Mourits clinical activity score (37), with 10 representing the most active disease and 0 the least active disease. 
isotype IgG control PE mouse antibody (BD, Ref 345816) or $5 \mu \mathrm{l}$ of anti-ICAM1 PE antibody (BD, Ref 555511), vortexed and incubated in darkness at $4{ }^{\circ} \mathrm{C}$ for $30 \mathrm{~min}$. OFs were washed with phosphate buffered azide and resuspended in $1 \%$ paraformaldehyde. The OFs were analyzed using a BD FACScan cytometer and Cellquest analysis software with data expressed as median fluorescent intensity of each experimental condition. Inter- and intra-assay coefficient of variation (CV) values were 2.7 and $2.2 \%$ respectively.

\section{GAG measurements}

The level of hyaluronic acid (HA) in the supernatants from the 48-h stimulation experiments described earlier was measured using an ELISA kit (K-1200, Echelon Biosciences, Inc., Salt Lake City, UT, USA) as per the manufacturer's instructions. Samples were diluted 1/10 before analysis, and the average of duplicate measurements was taken. Inter- and intra-assay $\mathrm{CV}$ values were 10.8 and $6.6 \%$ respectively.

\section{Adipogenesis}

Orbital fibroblasts were exposed to a differentiation protocol to encourage adipogenesis according to published methods $(12,19-22)$. OFs were grown to confluence in 6- or 12-well plates and exposed to differentiation media for 4 days (RPMI as detailed earlier, but lacking fetal bovine serum, and supplemented with $0.1 \mathrm{mM}$ IBMX, $1 \mu \mathrm{M}$ dexamethasone, $1 \mu \mathrm{M}$ rosiglitazone (Avandia, GSK), $100 \mathrm{nM}$ insulin (Actrapid, Novo Nordisk), $10 \mu \mathrm{g} / \mathrm{ml}$ transferrin (Sigma T 3309), $0.2 \mathrm{nM}$ T3 triiododo-L-thyronine (Fluka 91990). Media was replaced at days 4 and 8 with propagation media (identical to differentiation media but lacking the IBMX, dexamethasone and rosiglitazone) and cells analyzed at day 10. Cytokines and anticytokine agents were added at day 1 , and continued for the 10 day differentiation protocol, being replaced whenever media was replaced. Cells were stained for lipid using oil-red-O (ORO), photographed, and assessed for degree of adipogenesis using three methods: (i) extraction of ORO and measuring absorbance units (AU) at $492 \mathrm{~nm}$ (23), (ii) a visual scoring system (Table 2), and (iii) computer analysis of digital photographs.

\section{ORO staining and adipogenesis quantification}

ORO (0.7 g, Sigma O 0625) was dissolved in $200 \mathrm{ml}$ isopropanol (Sigma I 9030), shaken and left overnight at $21{ }^{\circ} \mathrm{C}$. The solution was repeatedly centrifuged at 13000 r.p.m. and decanted, until no un-dissolved debris remained. This solution was diluted 3:2 with distilled water and left overnight at $4{ }^{\circ} \mathrm{C}$. This $60 \%$ ORO solution was repeatedly centrifuged and decanted until no un-dissolved debris remained, and was then ready for use.

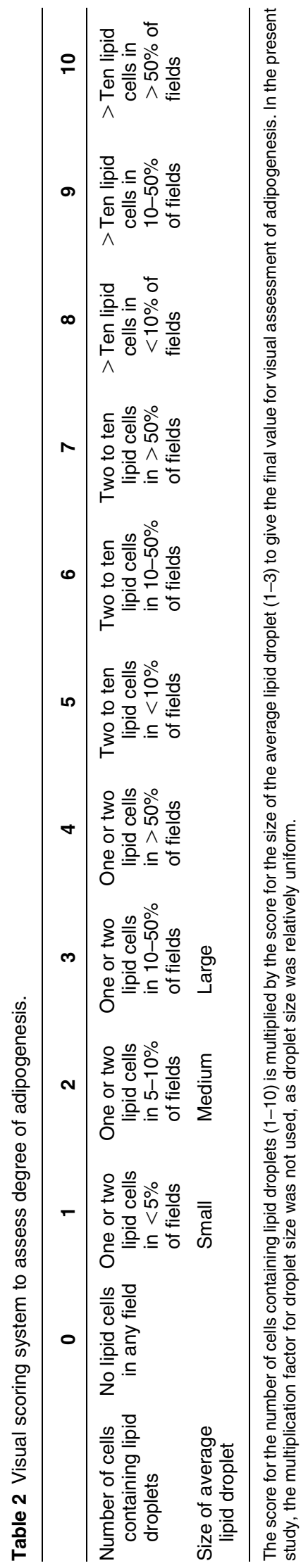

www.eje-online.org 
OFs ready for analysis were washed twice with PBS before being rinsed in $60 \%$ isopropanol for 30s. $200 \mu \mathrm{l}$ $60 \%$ ORO solution was added for $10 \mathrm{~min}$, before being removed and each well rinsed with $200 \mu \mathrm{l} 60 \%$ isopropanol for $10 \mathrm{~s}$. OFs were rinsed twice with PBS and photographed with a digital camera. The lipid droplets in the cells were stained orange-red and visually assessed for degree of adipogenesis. The wells were then washed four times with PBS to remove any remaining un-bound ORO. PBS was removed and $200 \mu \mathrm{l} 100 \%$ isopropanol was added for 5 min to extract the ORO. A volume of the supernatant $(170 \mu \mathrm{l})$ was transferred to a reading plate and absorbance units measured at $492 \mathrm{~nm}$. Since no individual well can be measured more than once, it was not possible to calculate inter- and intra-assay $\mathrm{CV}$ values for this method. However, in measuring wells containing cells from different patients, but under the same experimental conditions, the interand intra-assay variations (which includes inter-sample variation) were 26.9 and $8.5 \%$ respectively.

In order to assess adipogenesis according to the microscopy appearance of the OFs, a visual scoring system was designed (Table 2). This score was based upon the number of high-power $(200 \times)$ microscopy fields containing cells with lipid droplets, the number of cells per field containing lipid droplets and the average size of the lipid droplet (in the present study this multiplication factor for droplet size was not used as droplet size was relatively uniform). Approximately, ten fields in each well were examined, though more were viewed if there was uncertainty. The inter- and intra-assay $\mathrm{CV}$ values were 14.3 and $8.3 \%$ respectively. In addition, a microscopy analysis software package (AnalySIS, version 3.2, Soft Imaging Systems GmbH, Münster, Germany) was used to assess photographs of OFs stained with ORO taken by a digital camera mounted on a microscope. The software quantified the percentage of pixels in the photograph with a set degree of red, blue and green that corresponded to the areas of ORO staining. The inter- and intra-assay CV values were 21.3 and $1.7 \%$ respectively.

\section{Statistics}

The paired $t$-test was used to test ICAM, GAG and adipogenesis responses compared with the un-stimulated control condition from each experiment. Correlation of different methods of assessing adipogenesis was done using Pearson product moment correlation. Statistical significance was taken to be $<0.05$ and data analyzed using Minitab 13 software.

\section{Results}

\section{ICAM1 measurements; cytokine responses}

Experiments using different cell-detachment techniques (cell scraper, trypsin $(0.25 \%)$, trypsin $(0.25 \%) /$ EDTA
(1 $\mathrm{mM})$ and EDTA $(5 \mathrm{mM})$ ) prior to flow cytometry analysis found that the relative ICAM1 expression was similar across all methods, although the absolute measurements were $25-30 \%$ greater with trypsin/EDTA and EDTA. Trypsin $(0.25 \%)$ was used for this study. Dose-response curves were performed for each cytokine and where a dose-response was seen, concentrations either side of the ED50 were used on OFs from five patients with TAO and five control subjects. TNF $\alpha$, IL1 and IFN $\gamma$ stimulated ICAM1 expression in OFs from patients with TAO and control subjects in a similar, dose-dependent manner. TNF $\alpha$ $(0.1 \mathrm{ng} / \mathrm{ml})$, IL1 $(0.1 \mathrm{ng} / \mathrm{ml})$ and $\operatorname{IFN\gamma }(0.1 \mathrm{ng} / \mathrm{ml})$ stimulated ICAM1 expression eight- to ten-fold in OFs from patients with TAO ( $P$ values $<0.02)$. Interleukin$10(10 \mathrm{ng} / \mathrm{ml})$ and TGF $\beta(1 \mathrm{ng} / \mathrm{ml})$ had no effect. Nonspecific antibody binding as assessed by the non-specific isotype control antibody, remained unchanged throughout each experimental condition.

\section{Effect of anti-cytokine agents on cytokine responses}

The anti-TNF $\alpha$ agents adalimumab, etanercept and infliximab inhibited TNF $\alpha$-mediated ICAM1 expression in a dose-dependent manner. The IL1 receptor antagonist anakinra inhibited IL1-mediated ICAM1 expression in a dose-dependent manner. The anti-TNF $\alpha$ agents adalimumab, etanercept and infliximab inhibited the ICAM1 response to $0.1 \mathrm{ng} / \mathrm{ml} \mathrm{TNF} \alpha$ by $98 \%(51-144)$, 96\% (50-143) and 90\% (54-126) respectively, using peak concentrations, and 93\% (50-136), 81\% (53110 ) and $80 \%$ (58-104) respectively, using $1 / 100$ of peak concentrations (data expressed as mean $(95 \%$ Confidence interval (CI)), $P$ values $<0.005$; Fig. 1 ). The IL1 receptor antagonist anakinra inhibited the ICAM1 response to $0.1 \mathrm{ng} / \mathrm{ml}$ IL1 by $99 \%$ (78-119) using peak concentration and $69 \%(30-109)$ when using $1 / 100$ of the same $(P$ values $<0.01)$. The effect of the anticytokine agents was to bring the levels of ICAM1 expression close to, or back to, baseline levels (Fig. 1).

\section{HA measurements}

OFs from seven TAO patients and four control subjects showed similar HA responses to TNF $\alpha$, IL1 and their blockade (Fig. 1). In OFs from TAO patients, both TNF $\alpha$ $(0.1 \mathrm{ng} / \mathrm{ml})$ and IL1 $(0.1 \mathrm{ng} / \mathrm{ml})$ resulted in an increase in HA production compared with the basal un-stimulated condition (for $\mathrm{TNF} \alpha+938 \mathrm{ng} / \mathrm{ml}(+44 \%), 95 \% \mathrm{CI}$ $379-1498, P=0.006$ and for IL1 $+2016 \mathrm{ng} / \mathrm{ml}(+$ 95\%), 95\% CI 1029-3003, $P=0.002$; Fig. 1 ).

The reduction in TNF $\alpha$-stimulated HA production with etanercept $(2.5 \mu \mathrm{g} / \mathrm{ml})$ was $-1231 \mathrm{ng} / \mathrm{ml}(95 \%$ CI -362 to $-2099, P=0.013)$. The reduction in IL1stimulated HA production with anakinra $(5 \mu \mathrm{g} / \mathrm{ml})$ was $-1598 \mathrm{ng} / \mathrm{ml}(95 \% \mathrm{CI}-202$ to $-2994, P=0.031)$. This represented an inhibition of cytokine-mediated HA 


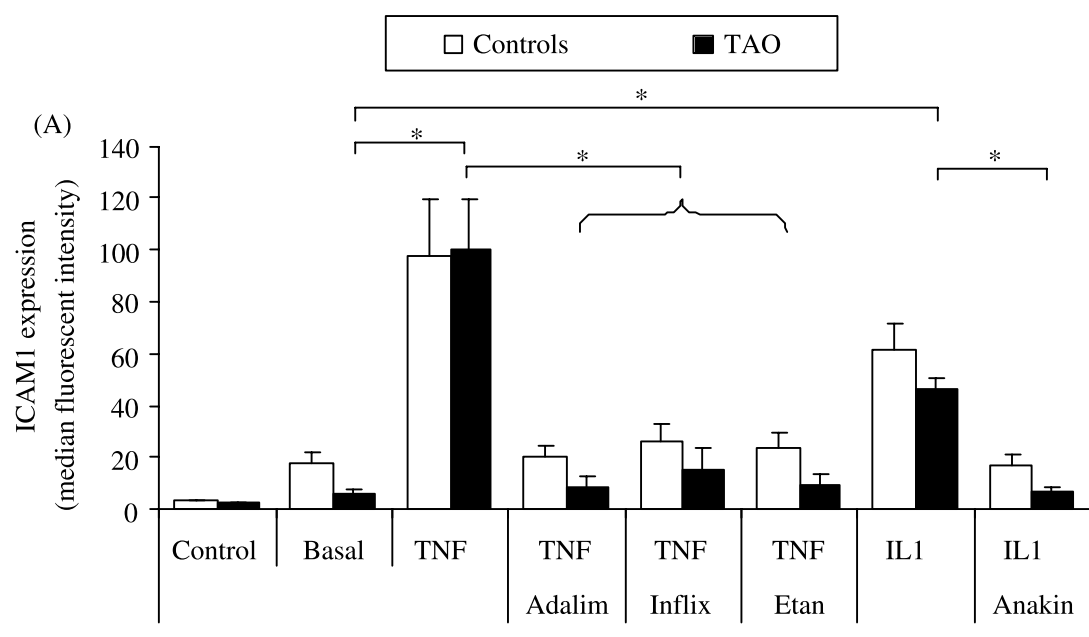

Figure 1 (A) The effect of anti-cytokine agents adalimumab $(50 \mu \mathrm{g} / \mathrm{ml})$, infliximab $(150 \mu \mathrm{g} / \mathrm{ml})$, etanercept $(2.5 \mu \mathrm{g} / \mathrm{ml})$ and anakinra $(5 \mu \mathrm{g} / \mathrm{ml})$ on TNF $\alpha(0.1 \mathrm{ng} / \mathrm{ml})$ or IL1 $(0.1 \mathrm{ng} / \mathrm{ml})$-mediated ICAM1 expression in OFs from patients with and without TAO ( $n=5$ in each group). Data expressed as mean \pm s.E.M. of median fluorescent intensities, expressed in arbitrary units. All cytokine-mediated increases in ICAM1 expression and their blockade by

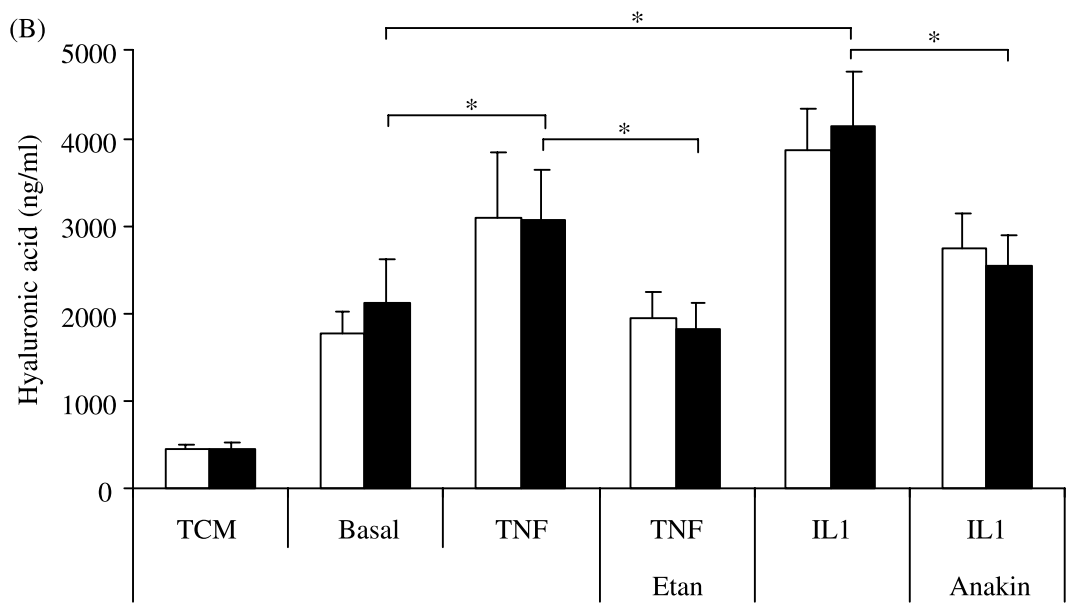
the anti-cytokine agent shown are statistically significant $\left({ }^{\star} P\right.$ value $\left.<0.01\right)$. Control lane represents the isotype control antibody and basal represents the measurement from OFs in the absence of cytokine stimulation. (B) Effect of anti-cytokine agents etanercept $(2.5 \mu \mathrm{g} / \mathrm{ml})$ and anakinra $(5 \mu \mathrm{g} / \mathrm{ml})$ on TNF $\alpha(0.1 \mathrm{ng} / \mathrm{ml})$ or IL1 $(0.1 \mathrm{ng} / \mathrm{ml})$-mediated in hyaluronic acid (HA) production by OFs from patients with TAO $(n=7)$ and control patients $(n=4)$. Data expressed as mean \pm S.E.M. In the TAO group, the cytokine-mediated increases in HA production and their blockade by the anti-cytokine agent shown are statistically significant $\left({ }^{*} P\right.$ value $<0.02)$. TCM represents the tissue culture medium used in each experiment, unexposed to OFs.

production of $138 \%$ (95\% CI 38-224) for etanercept and $79 \%$ (95\% CI 10-149) for anakinra. The effect of the anti-cytokine agents was to bring the HA levels close to, or back to, baseline levels (Fig. 1).

\section{Adipogenesis}

The adipogenic media caused accumulation of lipid droplets in a proportion of OFs, visible from day 3, which increased in number and enlarged in size during the subsequent 7 days of the differentiation protocol. The ability of OFs to accumulate lipid generally decreased with increasing cell passage, so each 6- or 12-well plate contained cells from the same patient who had undergone the same number of passages, and each plate had its own control well. The mean passage number was 3 (range 1-6), and similar responses were seen in cells from patients with or without TAO.

The ORO extraction data and the visual scoring system had a Pearson correlation coefficient of 0.89 , $P=0.017, R^{2}=0.79$ (average data from 6 plates). The
ORO extraction data and the data from computer analysis of digital photographs had a Pearson correlation coefficient of $0.84, P=0.038, R^{2}=0.70$ (average data from five plates).

$\mathrm{TNF} \alpha(0.1 \mathrm{ng} / \mathrm{ml})$ had an inhibitory effect on adipogenesis when measured by ORO extraction $(-0.013 \mathrm{AU}$, $95 \% \mathrm{CI}-0.024$ to $-0.002, P=0.025, n=8)$ and visual assessment $(-1.0,95 \% \mathrm{CI}-1.6$ to $-0.38, P=0.007$, $n=8)$. The inhibitory effect of TNF $\alpha$ was not significant when assessed by computer analysis of digital photographs $(-0.02 \%, 95 \%$ CI $0.1-0.07, P=0.7, n=8)$, but by this method, the TNF $\alpha$ response was significantly different from the IL1 $(0.1 \mathrm{ng} / \mathrm{ml})$ response (difference of $0.56 \%, 95 \%$ CI $0.18-0.94, P=0.01, n=8$ ) (Figs 2-4). The inhibitory effect of TNF $\alpha$ was attenuated by the anti$\mathrm{TNF} \alpha$ agent etanercept, as measured by visual assessment in 12-well plate experiments (Fig. 3), but this did not reach statistical significance (difference $=0.56,95 \%$ CI -0.05 to $1.12, P=0.065)$.

IL1 $(0.1 \mathrm{ng} / \mathrm{ml})$ had a stimulatory effect on adipogenesis when measured by ORO extraction $(+$ 0.050 AU, 95\% CI 0.012-0.088), $P=0.017, n=8)$, 


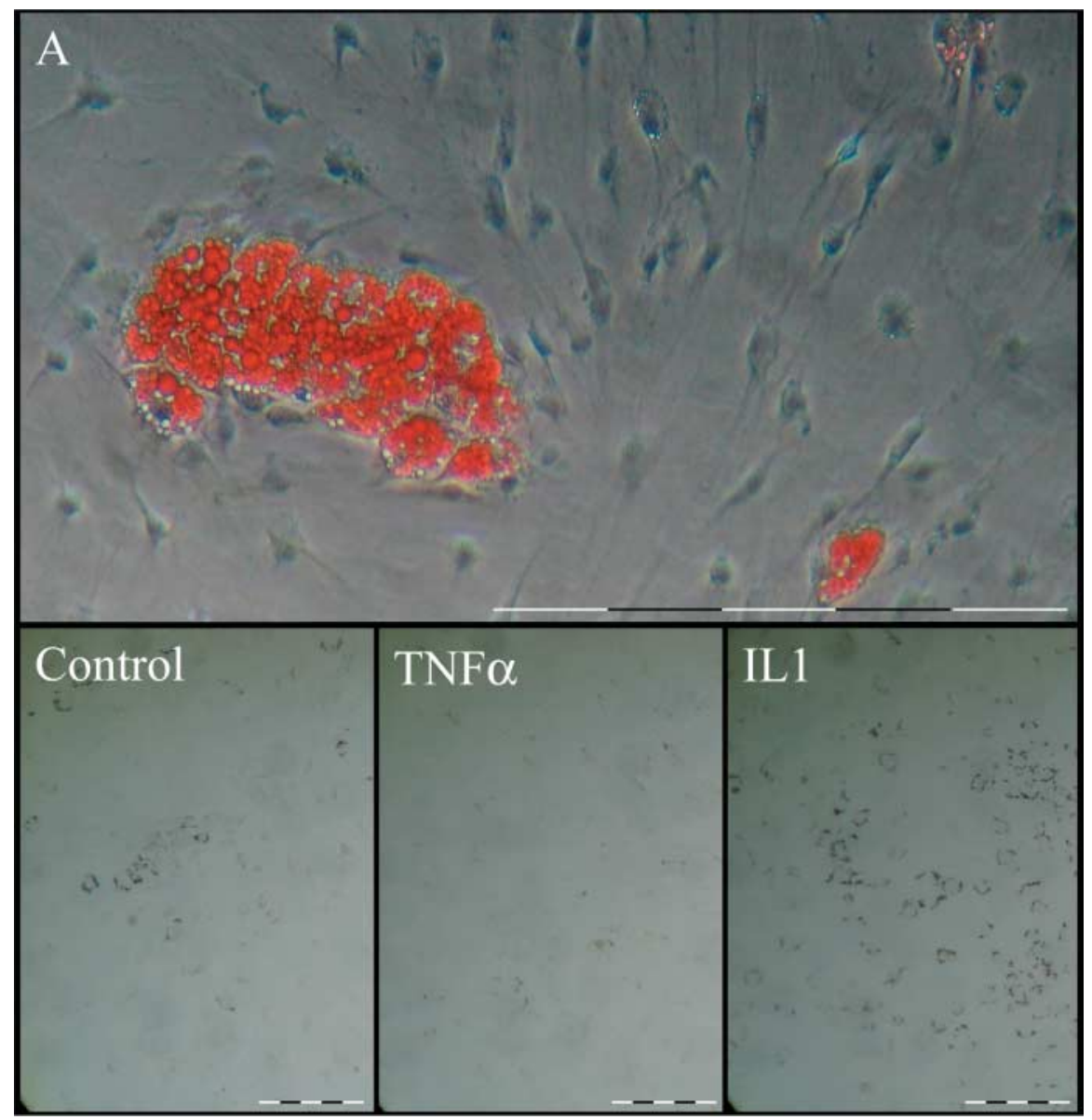

Figure 2 Examples of adipogenesis in orbital fibroblasts from a patient with TAO. Photographs were taken with a digital camera mounted on a light microscope and lipid is stained red with oil-red-O (ORO). (A) Highpower $(350 \times)$ example of localized adipogenesis, the other panels are low-power $(40 \times)$ photographs of orbital fibroblasts stained with ORO, with the control panel being of un-stimulated cells grown in adipogenic media for 10 days, the TNF panel being TNF $\alpha(0.1 \mathrm{ng} / \mathrm{ml})$ in adipogenic media, and the IL1 panel being IL1 $(0.1 \mathrm{ng} / \mathrm{ml})$ in adipogenic media. Total length of scale bar represents $500 \mu \mathrm{m}$. visual assessment $(+5.7,95 \%$ CI 3.9-7.5, $P<0.001)$ and computer analysis of digital photographs (+ $0.54 \%, 95 \%$ CI $0.20-0.87, P=0.007)$. Anakinra inhibited IL1-mediated adipogenesis measured by
ORO extraction $(-0.043 \mathrm{AU}, 95 \% \mathrm{CI}-0.080$ to $-0.005, P=0.034, n=8)$, visual assessment $(-4.0$, $95 \%$ CI -4.8 to $-3.2, P<0.001)$ and computer analysis of digital photographs $(-0.57 \%, 95 \%$ CI

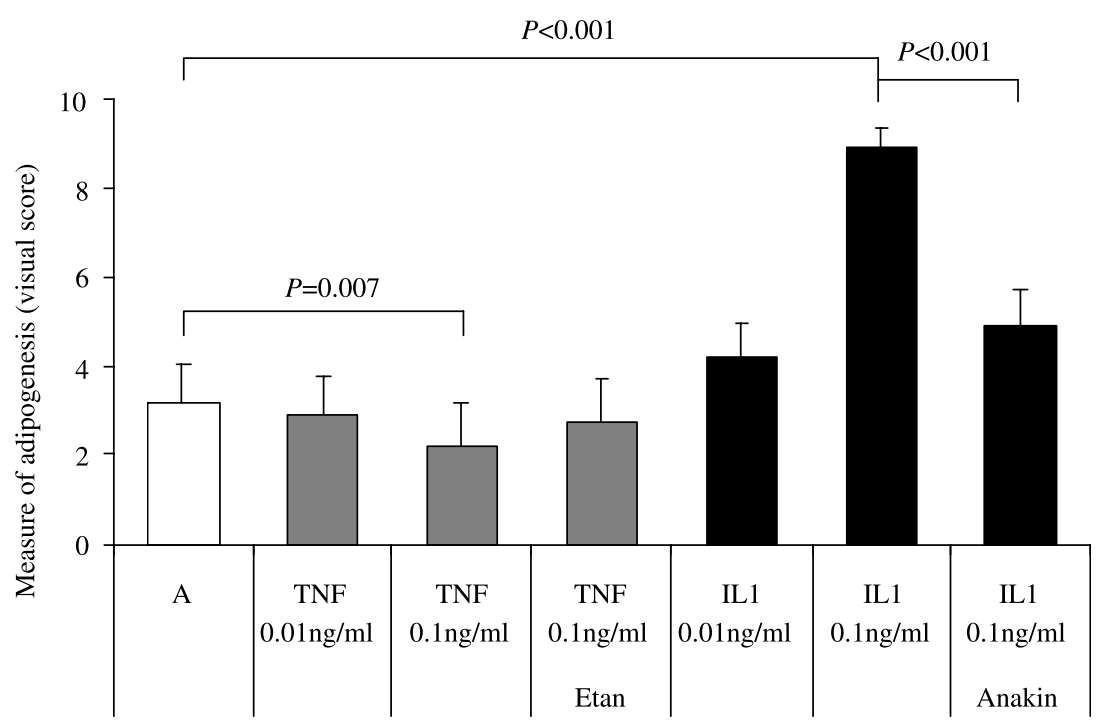

Figure 3 Effects of TNF $\alpha$ and IL1 and their antagonists (etanercept $2.5 \mu \mathrm{g} / \mathrm{ml}$ and anakinra $5.0 \mu \mathrm{g} / \mathrm{ml}$ ) on adipogenesis, expressed as mean \pm s.E.M., measured by visual assessment of microscopy appearance. A value of zero represents no adipogenesis and 30 represents maximal possible adipogenesis. Cells were grown in 12 -well plates. The value labeled ' $A$ ' represents the un-stimulated well that contained adipogenic media but no cytokines. 


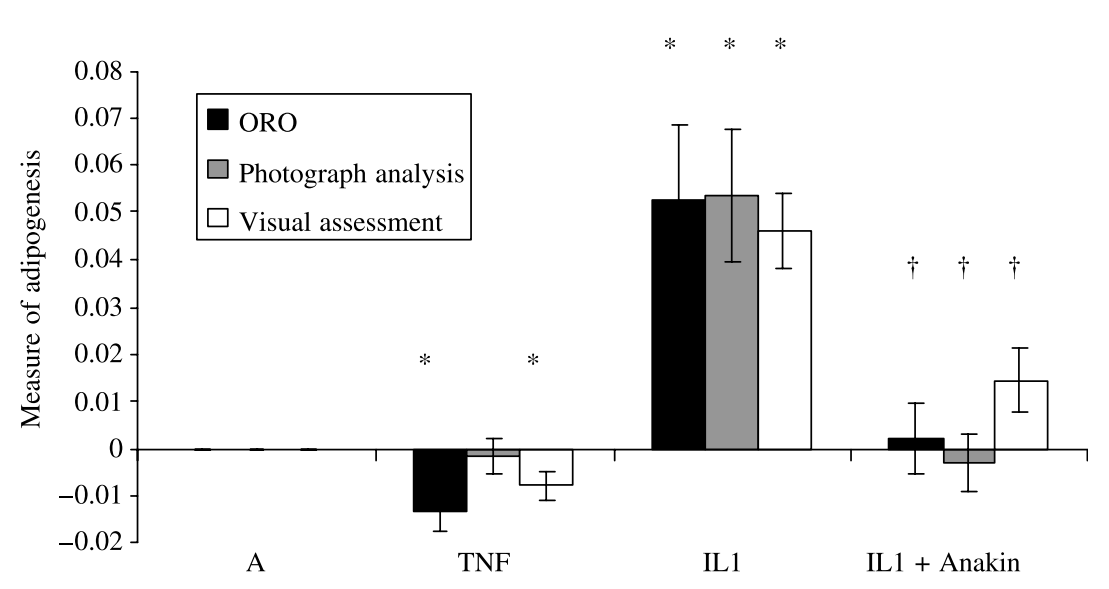

Figure 4 Effects of TNF $\alpha(0.1 \mathrm{ng} / \mathrm{ml}), \mathrm{IL} 1$ $(0.1 \mathrm{ng} / \mathrm{ml}) \pm$ anakinra $(5 \mu \mathrm{g} / \mathrm{ml})$ on adipogenesis, expressed as mean \pm S.E.M., as measured by oil-red-O (ORO) extraction (absorbance units at $492 \mathrm{~nm} ; n=8$ ), computer analysis of digital photographs (percentage of adipogenic pixels per photograph, $n=$ 8 ), and by visual assessment (scale of $0-30$, $n=8$ ), compared with the un-stimulated well (A) that contained adipogenic media but no cytokines. Cells were grown in six-well plates. The photograph analysis data have been adjusted (multiplied by 0.1 ) and visual assessment data have been adjusted (multiplied by 0.008 ) in order to present on same $y$-axis scale as ORO extraction. *Statistically significant difference from baseline $(P<0.05)$, and ${ }^{\dagger}$ statistically significant difference from corresponding IL1stimulated condition $(P<0.05)$.
-0.89 to $-0.25, P=0.004$ ) (Figs 3 and 4 ). This represented an inhibition of IL1-mediated adipogenesis of 96,69 and $106 \%$, measured by ORO extraction, visual assessment and computer analysis of digital photographs respectively. The stimulatory effect of IL1 persisted in the absence of peroxisome proliferatoractivated receptor $\gamma(\operatorname{PPAR} \gamma)$ agonist, though the degree of adipogenesis was reduced across all conditions (data not shown).

\section{Discussion}

This study has shown that TNF $\alpha$ and IL1 have stimulatory effects on ICAM1 expression and GAG production, but opposite effects on adipogenesis in OFs in vitro from patients with TAO. IL1 strongly promotes adipogenesis whilst TNF $\alpha$ has a weak inhibitory effect.

In the absence of an accepted animal model, we used the human model of TAO, which involves culturing OFs taken from patients undergoing surgery $(14,24)$. This model has certain limitations. The tissue obtained at surgery is often from patients whose disease is quiescent or, if done when the disease is active, these patients are usually already on immunosuppressive treatments, such as corticosteroids. The model only comprises fibroblasts in isolation, rather than in the presence of immunologically active cells such as lymphocytes and macrophages. Despite these obvious criticisms, this model is still widely used to study OF properties.

We did not observe obvious differences, either between the behaviour of OFs from patients with or without TAO, or between OFs from patients who had or had not received corticosteroid treatment for TAO. However, our study was not designed to detect small differences between these groups. If OFs from both TAO patients and healthy controls do indeed behave in a similar way, it would suggest that it is the presence or the absence of key cytokines in the orbit that influences the occurrence of TAO, rather than the development being dependent upon inter-individual differences in OF properties.

ICAM1 is an adhesion molecule that promotes inflammatory cell recruitment when upregulated. ICAM1 has previously been shown to be present in histological sections or orbital tissue taken from patients with TAO (11, 25-27). Using immunocytochemistry IL1, TNF $\alpha$ and IFN $\gamma$ have been shown to increase ICAM1 expression on OFs in vitro (10). In this study, we used flow cytometry to examine ICAM1 expression as it is a quantitative technique that allows more detailed study of the dose-related effects of cytokines and anticytokine agents. The stimulatory effects of TNF $\alpha$ and IL1 on ICAM1 were substantially reduced using clinically available anti-cytokine agents in concentrations achieved therapeutically. These data support the use of anti-cytokine agents to inhibit the inflammatory response in active TAO.

HA is a non-sulphated GAG that is synthesized at the plasma membrane, by a family of HA synthase enzymes. The excessive production of GAG by OFs in response to inflammatory cytokines may be one of the central mechanisms by which the autoimmune response in TAO leads to orbital tissue expansion. Orbital fibroblasts have been shown to increase their production of GAG in response to certain cytokines, such as TNF $\alpha$ (8), IL1 (7$9,28), \operatorname{IFN} \gamma(6,8)$ and TGF $\beta(7)$. We found that both TNF $\alpha$ and IL1 increased HA synthesis by OFs and that these responses could by inhibited by anti-TNF $\alpha$ and anti-IL1 agents. These data support the use of anticytokine agents to inhibit GAG production and the consequent orbital tissue volume expansion in active TAO.

In addition to increased GAG production, the other main mechanism of orbital tissue volume expansion in $\mathrm{TAO}$ is adipogenesis. It has been shown in vitro that a 
proportion of OFs have the ability to differentiate into adipocytes under certain conditions $(12,19-21)$. There is evidence of overexpression of mature adipocyterelated genes such as leptin, adiponectin and PPAR $\gamma$ and cysteine-rich angiogenic inducer 61 in TAO compared with normal orbital tissue samples $(29,30)$. This supports the concept that de novo adipogenesis occurs within the orbit in active TAO.

Adipogenesis in vitro can be challenging to quantify accurately. The technique of ORO extraction is accepted and objective (23) but prone to error, particularly when the degree of adipogenesis is low due to the influence of unbound ORO. Simple visual assessment is commonly used to measure adipogenesis $(12,31)$. We developed a visual scoring system incorporating the number of microscopy fields containing OFs with lipid droplets, the number of cells per field containing lipid droplets and the average size of the lipid droplet. As with any other visual scoring system, it is liable to operator bias and sampling error. To reduce the influence of any operator bias we also utilized a microscopy software package system to analyse digital photographs of OFs stained with ORO. This method provided an additional objective measure of adipogenesis that correlated well with the ORO extraction and visual assessment techniques. We therefore consider that these techniques, whilst individually liable to certain errors, together are able to quantify reliably the effects of the cytokine.

Consistent with a previous study (12), we found $\mathrm{TNF} \alpha$ has an inhibitory effect on adipogenesis in OFs. In our hands, the magnitude of this effect was modest, only reaching statistical significance in two out of the three methods used for quantification. This may be partly due to the relatively low levels of baseline adipogenesis that we achieved with our protocol omitting certain additives, such as carbaprostacyclin and included lower concentrations of adipogenic additives than some other studies (22). If the in vivo effect of TNF $\alpha$ on inhibiting adipogenesis is important in TAO, then blocking TNF $\alpha$ might reduce this inhibitory influence and so encourage the expansion of orbital fat. This could potentially worsen the signs and symptoms of TAO. Such an effect would be countered by the likely beneficial effects on ICAM1 expression, inflammation and GAG production, but the balance of these effects could be detrimental, particularly where adipogenesis is predominant in the disease process. It should also be considered that high-dose steroids and agents, which influence arachidonic acid metabolism, are used to promote adipogenesis in vitro, and also used as antiinflammatory treatments for active TAO. This might mean that the pro-adipogenic properties of these agents (and possibly also anti-TNF $\alpha$ agents) are not clinically significant. However, the opposite view would be that the reason why the existing treatments for TAO are sometimes not as effective as desired is because of their harmful pro-adipogenic effects countering their beneficial anti-inflammatory effects.
In contrast, we found that IL1 had a strong stimulatory effect on adipogenesis. This may have important clinical implications. As IL1 promoted all three pathological aspects of the disease process studied, namely a marker of the acute inflammatory response, GAG production and adipogenesis, IL1 presents an attractive therapeutic target in TAO. Anti-IL1 agents may be the preferred anti-cytokine agent for clinical trials in active TAO both in terms of their potential to interrupt the disease processes and in terms of safety. The IL1 antagonist presently available in clinical practice (anakinra) poses many of the expected safety issues associated with an anti-cytokine agent. Nevertheless, it appears to have a more favourable safety profile compared with the anti-TNF $\alpha$ agents in terms of granulomatous infections, such as tuberculosis (32).

There have, as yet been no randomized, placebocontrolled trials of anti-cytokine agents in TAO, but a case series and other case reports are emerging $(33,34)$. These reports have used anti-TNF $\alpha$ agents and have generally been positive. However, the history of new treatments for TAO is sometimes of initial excitement (35), followed by an inability to repeat these positive results in larger, controlled trials (36). This may partly be due to the positive publication bias, and the fact that TAO improves spontaneously to a significant degree.

In conclusion, this study, along with others, points towards anti-TNF $\alpha$ agents having the potential to improve inflammation and reduce GAG production in TAO, but these beneficial effects might be counteracted by a possible worsening of any adipogenic component of the disease. In contrast, the anti-IL1 agents appear to have the potential to improve inflammation, reduce GAG production and inhibit adipogenesis, and hence could have a beneficial effect in active TAO.

\section{Acknowledgements}

This work was supported by the Health Research Board, Ireland, the Royal College of Physicians and Surgeons of Glasgow, UK and the Irish Endocrine Society.

\section{References}

1 Bahn RS \& Heufelder AE. Pathogenesis of Graves' ophthalmopathy. New England Journal of Medicine 1993329 1468-1475.

2 Bartalena L, Pinchera A \& Marcocci C. Management of Graves' ophthalmopathy: reality and perspectives. Endocrine Reviews 2000 21 168-199.

3 Cawood T, Moriarty P \& O'Shea D. Recent developments in thyroid eye disease. British Medical Journal 2004329 385-390.

4 Bartalena L, Marcocci C \& Pinchera A. Cytokine antagonists: new ideas for the management of Graves' ophthalmopathy. Journal of Clinical Endocrinology and Metabolism $1996 \mathbf{8 1} 446-448$.

5 Bahn RS. Cytokines in thyroid eye disease: potential for anticytokine therapy. Thyroid $1998 \mathbf{8} 415-418$. 
6 Smith TJ, Bahn RS, Gorman CA \& Cheavens M. Stimulation of glycosaminoglycan accumulation by interferon gamma in cultured human retroocular fibroblasts. Journal of Clinical Endocrinology and Metabolism 199172 1169-1171.

7 Korducki JM, Loftus SJ \& Bahn RS. Stimulation of glycosaminoglycan production in cultured human retroocular fibroblasts. Investigative Ophthalmology and Visual Science 199233 2037-2042.

8 Metcalfe RA \& Weetman AP. Stimulation of extraocular muscle fibroblasts by cytokines and hypoxia: possible role in thyroidassociated ophthalmopathy. Clinical Endocrinology $1994 \mathbf{4 0}$ $67-72$.

9 Tan GH, Dutton CM \& Bahn RS. Interleukin-1 (IL-1) receptor antagonist and soluble IL-1 receptor inhibit IL-1-induced glycosaminoglycan production in cultured human orbital fibroblasts from patients with Graves' ophthalmopathy. Journal of Clinical Endocrinology and Metabolism 199681 449-452.

10 Heufelder AE \& Bahn RS. Graves' immunoglobulins and cytokines stimulate the expression of intercellular adhesion molecule-1 (ICAM-1) in cultured Graves' orbital fibroblasts. European Journal of Clinical Investigation 199222 529-537.

11 Pappa A, Calder V, Fells P \& Lightman S. Adhesion molecule expression in vivo on extraocular muscles (EOM) in thyroidassociated ophthalmopathy (TAO). Clinical and Experimental Immunology $1997 \mathbf{1 0 8} 309-313$.

12 Valyasevi RW, Jyonouchi SC, Dutton CM, Munsakul N \& Bahn RS. Effect of tumor necrosis factor-alpha, interferon-gamma, and transforming growth factor-beta on adipogenesis and expression of thyrotropin receptor in human orbital preadipocyte fibroblasts. Journal of Clinical Endocrinology and Metabolism $2001 \mathbf{8 6}$ 903-908.

13 Prabhakar BS, Bahn RS \& Smith TJ. Current perspective on the pathogenesis of Graves' disease and ophthalmopathy. Endocrine Reviews 200324 802-835.

14 Bahn RS, Gorman CA, Woloschak GE, David CS, Johnson PM \& Johnson CM. Human retroocular fibroblasts in vitro: a model for the study of Graves' ophthalmopathy. Journal of Clinical Endocrinology and Metabolism 198765 665-670.

15 den Broeder A, van de Putte L, Rau R, Schattenkirchner M, Van Riel P, Sander O, Binder C, Fenner H, Bankmann Y, Velagapudi R, Kempeni J \& Kupper H. A single dose, placebo controlled study of the fully human anti-tumor necrosis factor-alpha antibody adalimumab (D2E7) in patients with rheumatoid arthritis. Journal of Rheumatology $2002292288-2298$.

16 Jatoi A, Jett JR, Sloan J, Novotny P, Ford J, Prabhakar U \& Loprinzi CL. A pilot study on safety and pharmacokinetics of infliximab for the cancer anorexia/weight loss syndrome in non-small-cell lung cancer patients. Supportive Care in Cancer 200412 859-863.

17 Lee H, Kimko HC, Rogge M, Wang D, Nestorov I \& Peck CC. Population pharmacokinetic and pharmacodynamic modeling of etanercept using logistic regression analysis. Clinical Pharmacology and Therapeutics 200373 348-365.

18 Chang DM, Chang SY, Yeh MK \& Lai JH. The pharmacokinetics of interleukin-1 receptor antagonist in Chinese subjects with rheumatoid arthritis. Pharmacological Research $200450371-376$.

19 Sorisky A, Pardasani D, Gagnon A \& Smith TJ. Evidence of adipocyte differentiation in human orbital fibroblasts in primary culture. Journal of Clinical Endocrinology and Metabolism $1996 \mathbf{8 1}$ 3428-3431.

20 Valyasevi RW, Erickson DZ, Harteneck DA, Dutton CM, Heufelder AE, Jyonouchi SC \& Bahn RS. Differentiation of human orbital preadipocyte fibroblasts induces expression of functional thyrotropin receptor. Journal of Clinical Endocrinology and Metabolism $1999 \mathbf{8 4} 2557-2562$.

21 Valyasevi RW, Harteneck DA, Dutton CM \& Bahn RS. Stimulation of adipogenesis, peroxisome proliferator-activated receptorgamma $(\operatorname{PPAR} \gamma)$, and thyrotropin receptor by PPAR $\gamma$ agonist in human orbital preadipocyte fibroblasts. Journal of Clinical Endocrinology and Metabolism 200287 2352-2358.

22 Hemmrich K, von Heimburg D, Cierpka K, Haydarlioglu S \& Pallua N. Optimization of the differentiation of human preadipocytes in vitro. Differentiation 200573 28-35.

23 Ramirez-Zacarias JL, Castro-Munozledo F \& Kuri-Harcuch W. Quantitation of adipose conversion and triglycerides by staining intracytoplasmic lipids with Oil red O. Histochemistry 199297 493-497.

24 Bahn RS. The fibroblast is the target cell in the connective tissue manifestations of Graves' disease. International Archives of Allergy and Immunology 1995106 213-218.

25 Heufelder AE \& Bahn RS. Elevated expression in situ of selectin and immunoglobulin superfamily type adhesion molecules in retroocular connective tissues from patients with Graves' ophthalmopathy. Clinical and Experimental Immunology 199391 381-389.

26 Kahaly G, Hansen C, Felke B \& Dienes HP. Immunohistochemical staining of retrobulbar adipose tissue in Graves' ophthalmopathy. Clinical Immunology and Immunopathology 199473 53-62.

27 He W, Luo Q \& Cheng N. A study of adhesion molecule expression in extraocular muscles of Graves' ophthalmopathy. Chung Hua Yen Ko Tsa Chih 200137 267-269.

28 Lu R, Wang P, Wartofsky L, Sutton BD, Zweier JL, Bahn RS, Garrity J \& Burman KD. Oxygen free radicals in interleukin-1beta-induced glycosaminoglycan production by retro-ocular fibroblasts from normal subjects and Graves' ophthalmopathy patients. Thyroid 19999 297-303.

29 Kumar S, Coenen MJ, Scherer PE \& Bahn RS. Evidence for enhanced adipogenesis in the orbits of patients with graves' ophthalmopathy. Journal of Clinical Endocrinology and Metabolism 200489 930-935.

30 Lantz M, Vondrichova T, Parikh H, Frenander C, Ridderstrale M, Asman P, Aberg M, Groop L \& Hallengren B. Overexpression of immediate early genes in active Graves' ophthalmopathy. Journal of Clinical Endocrinology and Metabolism 200590 4784-4791.

31 Permana PA, Nair S, Lee YH, Luczy-Bachman G, Vozarova De Courten B \& Tataranni PA. Subcutaneous abdominal preadipocyte differentiation in vitro inversely correlates with central obesity. American Journal of Physiology. Endocrinology and Metabolism 2004 286 E958-E962.

32 Fleischmann R, Stern R \& Iqbal I. Anakinra: an inhibitor of IL-1 for the treatment of rheumatoid arthritis. Expert Opinion on Biological Therapy 20044 1333-1344.

33 Paridaens D, van den Bosch WA, van der Loos TL, Krenning EP \& van Hagen PM. The effect of etanercept on Graves' ophthalmopathy: a pilot study. Eye 200519 1286-1289.

34 Durrani OM, Reuser TQ \& Murray PI. Infliximab: a novel treatment for sight-threatening thyroid associated ophthalmopathy. Orbit 200524 117-119.

35 Krassas GE, Kaltsas T, Dumas A, Pontikides N \& Tolis G. Lanreotide in the treatment of patients with thyroid eye disease. European Journal of Endocrinology 1997136 416-422.

36 Dickinson AJ, Vaidya B, Miller M, Coulthard A, Perros P, Baister E, Andrews CD, Hesse L, Heverhagen JT, Heufelder AE \& KendallTaylor P. Double-blind, placebo-controlled trial of octreotide longacting repeatable (LAR) in thyroid-associated ophthalmopathy. Journal of Clinical Endocrinology and Metabolism $2004 \mathbf{8 9}$ 5910-5915.

37 Mourits MP, Prummel MF, Wiersinga WM \& Koornneef L. Clinical activity score as a guide in the management of patients with Graves' ophthalmopathy. Clinical Endocrinology (Oxf) 1997 47 9-14.

Received 2 June 2006

Accepted 3 July 2006 